\title{
Meeting report microRNAs mature with help from cancer biology
}

\author{
Zain Paroo* and Alexander Pertsemlidis*†
}

Addresses: *Department of Biochemistry and tDepartment of Internal Medicine and Harold C Simmons Comprehensive Cancer Center, UT Southwestern Medical Center, Dallas, TX 75930-8591, USA.

Correspondence: Alexander Pertsemlidis. Email: Alexander.Pertsemlidis@UTSouthwestern.edu

\begin{abstract}
A report of the Keystone Symposium on Molecular and Cellular Biology, 'MicroRNA and Cancer', Keystone, Colorado, USA, 10-15 June 2009.
\end{abstract}

A recent meeting in the series of Keystone Symposia focused on the intersection of microRNA (miRNA) and cancer biology. The meeting was characterized by the traditional hallmarks of Keystone Symposia: a defined scientific focus, a single thread and an emphasis on presentation of unpublished results. The breadth of topics covered reflected the rapid maturation of the field. Here, we highlight three major themes that ran through the plenary sessions.

\section{Non-coding RNA variation}

Our understanding of the importance of non-coding RNA (ncRNA) biology has evolved rapidly. John Mattick (University of Queensland, Brisbane, Australia) reminded us that organismal complexity scales with the non-coding rather than the coding genome. These are still early days for the field, with new classes of ncRNA, such as transcription initiation RNA (tiRNA), and new functions for understudied ncRNA, such as small nucleolar RNA (snoRNA), on the horizon. Highlighting important advances in our understanding of molecular biology, Mattick discussed dissociated expression of 3' untranslated regions (UTRs) from their coding sequences, which would enable genetic information to be transmitted in trans. Joel Neilson (Massachusetts Institute of Technology, Cambridge, USA) later picked up this thread and demonstrated dynamic regulation of 3' UTR expression. He described work on primary lymphocytes in which cellular proliferation was associated with reduced 3' UTR length, findings that parallel the decreased miRNA expression seen in carcinomas.

Joanne Weidhaas (Yale University School of Medicine, New Haven, USA) reported efforts to identify singlenucleotide polymorphisms (SNPs) in the miRNA tumor suppressor let-7. Although no genomic mutations in let-7 were identified, a SNP was identified in one of the let-7 target sites in the 3' UTR of the oncogene KRAS. Characterized in 2,433 people representing 46 human populations, this let-7 target SNP (or 'oncoSNP') was identified in up to $20.3 \%$ of non-small cell lung carcinoma (NSCLC) patients and $5.8 \%$ of the world population. It is also associated with $B R C A 1 / 2$-negative hereditary breast and ovarian cancer (HBOC), and a strong argument was made for the oncoSNP as a genetic marker of cancer risk.

Several presenters reported observations suggesting that expression of let-7 is differentially regulated relative to that of other miRNAs. Scott Hammond (University of North Carolina, Chapel Hill, USA) reported reduced processing of primary and precursor let-7 by Lin-28 in embryonic carcinoma cells. He noted work by George Daley and colleagues in which primary human tumors showed overexpression of lin-28, repression of let-7 and de-repression of let-7 targets. Eric Miska (University of Cambridge, UK) reported on complementary work demonstrating that in Caenorhabditis elegans, lin-28 forms a complex with the uridyl transferase PUP-2/TUT-4 and mediates degradation of pre-let-7.

A genetic explanation for the global reduction in miRNA expression that is a hallmark of cancer cells was offered by Sonia Melo (Institut d'Investigació Biomèdica de Bellvitge, Barcelona, Spain). When screening for exonic repeats in miRNA pathway components, Melo and colleagues found two frameshift mutations in the human Dicer protein cofactor TRBP (trans-activation-responsive RNA-binding protein). Both mutations introduced premature stop codons resulting in reduced TRBP expression. Because one function for TRBP is regulating Dicer stability, these mutations resulted in reduced Dicer expression and lower miRNA production and were associated with higher cellular proliferation.

Reporting on profiling the miRNA transcriptome of a murine leukemia cell line, Florian Kuchenbauer (Terry Fox Laboratory, Vancouver, Canada) discussed miRNA sequence variants (isomiRs) that could be due to differences in premiRNA biogenesis. He explored the role of miRNA* (putative passenger strand) sequences in expanding the 
target range of a miRNA duplex, proposing two classes of miRNA loci, those for which the miRNA/miRNA* ratio is maintained across cells and those for which it is dynamic.

\section{Prediction of miRNA targets}

Prediction of miRNA targets was clearly and repeatedly identified as a major challenge for the field. Despite numerous advances in experimental validation of miRNA targets and general agreement on the determinants of the interaction between miRNAs and their target sites (perfect complementarity in the seed sequence of the miRNA, a favorable free energy of hybridization and accessibility of the target site in the 3 ' UTR), commonly used packages for the prediction of miRNA target sites show poor overlap in their predictions. In parallel, two opposing views of miRNA regulation were presented, one holding that miRNAs are master regulators, with each miRNA governing one or a few 'important' targets, and the other proposing that miRNAs fine-tune the expression of many targets.

The rna22 algorithm designed by Isidore Rigoutsos (IBM Watson Research Center, Yorktown, USA) does not use seed complementarity as a constraint, permits bulges and G:U pairs in seed sequences and does not require conservation. Overall, it predicts an average of 1,00o targets per miRNA, with 15 miRNAs targeting the average mRNA, and indicates that more than $92 \%$ of genes are under miRNA control. Rigoutsos asserted that coding regions and 5' UTRs are valid regions in which miRNA target sites should be functional and showed confirmation of both conserved and non-conserved target sites in coding regions. Mark Kay (Stanford University School of Medicine, USA) offered an experimental explanation as to why most known miRNA target sites are located in the 3' UTR. He outlined a 'traffic jam' model in which ribosomal movement along the coding region of a transcript blocks access of the RNAinduced silencing complex (RISC) to the mRNA whereas rare codons slow translation and allow RISC access to the transcript. This model gives new potential relevance to the synonymous substitutions that have long failed to capture the attention of the genetics community.

Three strategies to facilitate experimental validation of miRNA targets were presented. First, Amy Pasquinelli (University of California, San Diego, USA) and Liang Zhang (Rockefeller University, New York, USA) reported approaches that couple immunoprecipitation of known miRNA factors and global analyses to identify functional miRNA and mRNA complexes. Known miRNA targets are enriched in immunoprecipitates that correlate with corresponding miRNAs. This combination of techniques identified novel miRNAs and many miRNA:mRNA target interactions, some with imperfect seed matches, but generally supporting the idea that perfect complementarity between bases 2-8 of the miRNA and the target are a real determinant of a functional interaction. Second, Michelle
Cleary (Rosetta Inpharmatics, Seattle, USA) underscored the idea that complementarity to the seed sequence of a miRNA cannot always be confirmed functionally. Enhanced effects of transfected miRNA mimics were observed in Dicer hypomorphic cells, allowing identification of several miRNAs associated with cell cycle progression and cellular response to DNA damage, including miR-34a. Third, Judy Lieberman (Harvard Medical School, Cambridge, USA) discussed identification of $m i R-24$ as a tumor suppressor. In response to $m i R-24$ overexpression, 248 mRNAs were downregulated. Pathway analysis led to the identification of several unpredicted targets, including those encoding the transcription factors Myc and E2F2. Seven of these interactions were based on 'seedless' target sites.

\section{miRNA-based therapeutics}

Therapeutics using miRNA have enormous but as yet unrealized potential. Tumor suppressor miRNA mimics and oligonucleotide inhibitors complementary to oncogenic miRNA can be used to restore normal cell programming. However, the 30-year history of nucleic acid drug development highlights many important challenges, including formulation and pharmacology. Josh Mendell (Johns Hopkins University, Baltimore, USA) and Frank Slack (Yale University) both reported on use of miRNA mimics in the context of replacement therapy. Mendell outlined the systemic delivery of $m i R-26 a$ by adenoassociated virus to induce tumor-specific apoptosis in mouse models of hepatocellular carcinoma through downregulation of cyclins D2 and E2. Slack reported remission of established NSCLC tumors in mice following exogenous delivery of a synthetic let-7 mimic.

Reports from two biopharmaceutical companies focused on miRNA therapeutics. Eric Marcusson (Regulus Therapeutics, Carlsbad, USA) discussed the potential benefits of single-stranded oligonucleotides, which do not require a delivery vehicle, relative to double-stranded miRNA mimics, which require formulation. The challenge for working with single-stranded oligonucleotides is in achieving a nucleic acid chemistry composition that is both a suitable pharmacological agent and compatible with the miRNA machinery. A single-stranded inhibitor of miR-122 for cholesterol regulation and a double-stranded mimic of tumor suppressor miR-34a in cationic lipid and liposomal nanoparticle formulations were discussed. David Brown (Asuragen/miRNA Therapeutics, Austin, USA) also presented progress with a $m i R-34 a$ mimic using a non-charged lipid formulation. miRNA Therapeutics is focusing on miRNA replacement therapy rather than anti-miRNA approaches. Intravenous delivery of miR-Rx34 inhibited the growth and metastasis of established human tumors in mouse models of lung and prostate cancer. Remarkably, significant results were achieved with therapeutic doses as low as $10 \mu \mathrm{g} / \mathrm{kg}$ of animal body weight, more than two orders of 
magnitude lower than what has been reported by others, offering a great deal of promise for this class of therapeutic.

Overall, this second meeting was a milestone in the evolution of the field, marked by a change in emphasis from miRNA biology to cancer biology and a maturation from general questions of whether miRNA are differentially expressed in cancers to whether miRNAs are of therapeutic utility. As cancer biology has provided direction for investigations of miRNA biology, the discovery of miRNAs has also changed thinking in cancer research. Profiling of human tumors has identified signatures associated with risk, diagnosis, prognosis and therapeutic response. Reports of differential expression of highly conserved non-coding genes in malignant cells relative to normal cells are providing better understanding of disease progression. Correlation between genomic locations of miRNAs and cancer-associated genomic regions has coupled aberrant miRNA expression to the genetic basis of cancer. The expanded study of pathways relevant to cancer processes is leading to new insights on the cellular and epigenetic regulation of miRNA genes and miRNA pathway components. Taken together, these advances comprise a revisiting of molecular oncology dogma, characterized by George Calin (MD Anderson Cancer Center, Houston, USA) as a re-opening of Pandora's box, from which miRNAs and highly conserved non-coding genes emerge as principal candidates for cancer-predisposing genes. Genetics and biochemistry have been of crucial importance in developing the models and rules that define where the field is today. The next steps are clearly in the direction of systems biology, which will take us from investigations of single miRNA and single targets to more comprehensive explorations of the regulatory networks through which they interact and function.

Published: 20 July 2009

doi:10.1186/gb-2009-10-7-310

(c) 2009 BioMed Central Ltd 